UUITP-27/12

\title{
On the distribution of stable de Sitter vacua
}

\author{
Ulf Danielsson and Giuseppe Dibitetto \\ Institutionen för fysik och astronomi, \\ University of Uppsala, \\ Box 803, SE-751 08 Uppsala, Sweden \\ $\{$ ulf.danielsson, giuseppe.dibitetto\}@physics.uu.se
}

\begin{abstract}
The possible existence of (meta-) stable de Sitter vacua in string theory is of fundamental importance. So far, there are no fully stable solutions where all effects are under perturbative control. In this paper we investigate the presence of stable de Sitter vacua in type II string theory with non-geometric fluxes. We introduce a systematic method for solving the equations of motion at the origin of moduli space, by expressing the fluxes in terms of the supersymmetry breaking parameters. As a particular example, we revisit the geometric type IIA compactifications, and argue that non-geometric fluxes are necessary to have (isotropically) stable de Sitter solutions. We also analyse a class of type II compactifications with non-geometric fluxes, and study the distribution of (isotropically) stable de Sitter points in the parameter space. We do this through a random scan as well as through a complementary analysis of twodimensional slices of the parameter space. We find that the (isotropically) stable de Sitter vacua are surprisingly rare, and organise themselves into thin sheets at small values of the cosmological constant.
\end{abstract}




\section{Contents}

1 Introduction $\quad 1$

$2 \mathcal{N}=1$ compactifications with generalised fluxes $\quad 3$

2.1 The $\mathbb{Z}_{2} \times \mathbb{Z}_{2}$ orbifold . . . . . . . . . . . . . . . . . 3

2.2 A new systematic prescription . . . . . . . . . . . . . . 8

2.3 Geometric IIA backgrounds: an over-constrained example . . . . . . . . . . 9

3 Towards stable dS solutions 12

3.1 A type IIB setup with generalised fluxes . . . . . . . . . . . . . . . 13

3.2 Statistical scan and tests of RMT . . . . . . . . . . . . . . 13

3.3 Analysis of the stable $\mathrm{dS}$ region . . . . . . . . . . . . . . 16

4 Conclusions $\quad 18$

A All the solutions of type IIB with $F, H$ and $Q \quad 20$

\section{Introduction}

Since the turn of the millennium, there have been many attempts to embed dark energy and inflation in string theory using flux compactifications. Parallelly, a whole class of string theory constructions involving flux backgrounds compatible with minimal supersymmetry has been considered in the literature. In particular, the mechanism of inducing effective superpotentials from fluxes [1] has often been considered in the context of the supergravity descriptions normally referred to as $S T U$-models [2-10]. It has, however, turned out to be surprisingly difficult to find the required stable or quasi-stable de Sitter (dS) solutions through compactifications of string theory. Let us start with a brief review of the main results of these searches.

Based on the results of [11], in ref. [12] all the type IIA coset compactifications with $\mathrm{SU}(3)$ structure were analysed, and a no-go result was proven in all the cases but one. The only model evading these no-go theorems turned out to be an $\mathrm{SU}(2) \times \mathrm{SU}(2)$ compactification. This model was indeed numerically shown to contain a dS solution, which was found to be unstable due to the presence of a tachyon already within the isotropic subsector. This example of a type IIA unstable dS extremum was later studied in ref. [13] (see also [14]), where it was shown to belong to a whole continuous line of tachyonic solutions, for which the ten-dimensional uplift to massive IIA supergravity was constructed in terms of very simple 
torsion classes. This was accommodated in a general framework in [15]. For further analysis of this model, see ref. [16]. In parallel, in ref. [17], many type II setups in the $\mathbb{Z}_{2} \times \mathbb{Z}_{2}$ orbifold with generalised fluxes were analysed searching for dS solutions. In a setup corresponding to type IIA with O6/D6 and only geometric fluxes, a dS region was found in the neighbourhood of a Minkowski critical point, but again with no room for stability. Later in ref. [18], the original dS solution of ref. [12] was localised at a particular point within the aforementioned dS region.

Finally in ref. [19], the complete set of solutions in the framework of isotropic $\mathcal{N}=1$ geometric type IIA compactifications on $T^{6} /\left(\mathbb{Z}_{2} \times \mathbb{Z}_{2}\right)$ was presented. It turns out to consist of a discrete set of lines, of which only one crosses the Minkowski point [20] and enters the dS region, with tachyons at every point. This can be regarded as an indication that one needs to consider more general setups including non-geometric fluxes [21] in order to find stable dS vacua [17].

The main goal of this paper is to show how non-geometric fluxes indeed provide enough freedom in parameter space for tuning the masses of all the moduli to be positive. Moreover, in situations where this is the case, we would like to locate the region of stable dS in parameter space and qualitatively estimate which portion of the total volume this corresponds to.

The paper is organised as follows. In section 2 we introduce the $\mathbb{Z}_{2} \times \mathbb{Z}_{2}$ compactifications with orientifold and their superpotential formulations. Subsequently, we present a new technique for solving the equations of motion in the origin of moduli space. In such a framework it becomes straightforward to predict the dimensionality of the parameter space of solutions. Finally, we make use of this machinery to review type IIA compactifications with geometric fluxes regarded as an over-constrained particular sub-case. In section 3 a IIB setup with non-geometric fluxes is introduced in which the parameter space has the correct degrees of freedom to gain control of the masses for all the isotropic moduli. A statistical scan of the parameter space is performed, and the fraction of stable solutions is plotted as a function of the normalised cosmological constant. Furthermore, we show some two-dimensional slices of the parameter space, and locate the thin region of stable dS critical points. Finally, we present our conclusions. An appendix is provided where we give some details about the analytical solutions in type IIB with $F, H$ and $Q$ fluxes. 


\section{$2 \mathcal{N}=1$ compactifications with generalised fluxes}

\subsection{The $\mathbb{Z}_{2} \times \mathbb{Z}_{2}$ orbifold}

A wide class of string compactifications down to four dimensions compatible with minimal supersymmetry can be effectively described by particular supergravity theories which are known as $S T U$-models. These theories arise, e.g., from $T^{6} /\left(\mathbb{Z}_{2} \times \mathbb{Z}_{2}\right)$ orbifold compactifications of type IIB with O3/O7-planes (and duals thereof). This class of string compactifications has the very appealing feature of collecting together low-energy effective descriptions which are related to each other by dualities without the intervention of mirror symmetry. In the $\mathbb{Z}_{2} \times \mathbb{Z}_{2}$ orbifold any dual effective theory is still formally described by the same Lagrangian where only fields and couplings have been transformed.

This class of effective descriptions enjoys $\mathcal{N}=1$ supersymmetry and $\operatorname{SL}(2)^{7}$ global bosonic symmetry due to the coupling of the gravity multiplet with seven extra chiral multiplets. The scalar sector contains seven complex fields spanning the coset space $(\mathrm{SL}(2) / \mathrm{SO}(2))^{7}$ which we denote by $\Phi^{\alpha} \equiv\left(S, T_{i}, U_{i}\right)$ with $i=1,2,3$. In the type IIB language, the $S$ modulus contains the ten-dimensional dilaton, whereas the $T$ and $U$ moduli are interpreted as Kähler and complex structure moduli respectively ${ }^{1}$. The kinetic Lagrangian can be derived from the following Kähler potential

$$
K=-\log (-i(S-\bar{S}))-\sum_{i=1}^{3} \log \left(-i\left(T_{i}-\bar{T}_{i}\right)\right)-\sum_{i=1}^{3} \log \left(-i\left(U_{i}-\bar{U}_{i}\right)\right) .
$$

This yields

$$
\mathcal{L}_{\text {kin }}=\frac{\partial S \partial \bar{S}}{(-i(S-\bar{S}))^{2}}+\sum_{i=1}^{3}\left(\frac{\partial T_{i} \partial \bar{T}_{i}}{\left(-i\left(T_{i}-\bar{T}_{i}\right)\right)^{2}}+\frac{\partial U_{i} \partial \bar{U}_{i}}{\left(-i\left(U_{i}-\bar{U}_{i}\right)\right)^{2}}\right) .
$$

The presence of fluxes induces a scalar potential $V$ for the moduli fields which is given in terms of the above Kähler potential and a holomorphic superpotential $W$ by

$$
V=e^{K}\left(-3|W|^{2}+K^{\alpha \bar{\beta}} D_{\alpha} W D_{\bar{\beta}} \bar{W}\right),
$$

where $K^{\alpha \bar{\beta}}$ is the inverse Kähler metric and $D$ denotes the Kähler-covariant derivative.

As a first example of flux-induced deformation, let us consider the presence of gauge fluxes in the duality frame of type IIB with $\mathrm{O} 3$ and O7-planes which gives rise to the following superpotential

$$
W=\underbrace{P_{F}\left(U_{i}\right)}_{F \text { flux }}+\underbrace{P_{H}\left(U_{i}\right) S}_{H \text { flux }}
$$

\footnotetext{
${ }^{1}$ Please note that, in a type IIA language, the ten-dimensional dilaton would correspond to a combination of $S$ and $T$, whereas the Kähler and complex structure moduli would be interchanged.
} 
where $P_{F}$ and $P_{H}$ are cubic polynomials in the complex structure moduli given by

$$
\begin{aligned}
& P_{F}\left(U_{i}\right)=a_{0}-\sum_{i} a_{1}^{(i)} U_{i}+\sum_{i} a_{2}^{(i)} \frac{U_{1} U_{2} U_{3}}{U_{i}}-a_{3} U_{1} U_{2} U_{3} \\
& P_{H}\left(U_{i}\right)=-b_{0}+\sum_{i} b_{1}^{(i)} U_{i}-\sum_{i} b_{2}^{(i)} \frac{U_{1} U_{2} U_{3}}{U_{i}}+b_{3} U_{1} U_{2} U_{3} .
\end{aligned}
$$

The $\mathcal{N}=1$ supergravity defined by the above superpotential has a no-scale feature due to the absence of the moduli $T_{i}$. This implies that their real parts appear as completely flat directions in the scalar potential. The line of including some non-perturbative effects such as gaugino condensation [22] has been considered as a possible mechanism to further stabilise the Kähler moduli (see e.g. refs $[23,24]$ ).

Duality covariance arguments would suggest to complete the superpotential given in (2.4) with more effective couplings which are obtained by performing SL $(2)^{7}$ transformations. This gives rise to a generalised form of $W$ containing all the possible $2^{7}=128$ couplings from the constant one up to the $S T_{1} T_{2} T_{3} U_{1} U_{2} U_{3}$ term. Such a very general superpotential, though, will be non-geometric in any duality frame.

\section{The isotropic truncation}

For the sake of simplicity, in the main part of this paper we will restrict ourselves to the isotropic limit of the theories previously presented. This reduces the number of complex moduli down to three via

$$
T_{1}=T_{2}=T_{3} \equiv T \text { and } \quad U_{1}=U_{2}=U_{3} \equiv U
$$

which span the scalar coset $\left(\frac{\mathrm{SL}(2)}{\mathrm{SO}(2)}\right)^{3}$. As for the fluxes, taking the isotropic limit implies the following identifications

$$
a_{1}^{(1)}=a_{1}^{(2)}=a_{1}^{(3)} \equiv a_{1}
$$

and similarly for $a_{2}^{(i)}, b_{1}^{(i)}$ and $b_{2}^{(i)}$.

In the isotropic limit, the complete duality covariant superpotential contains 32 couplings going from the constant term up to the $S T^{3} U^{3}$ term. For completeness, we give here the full form of the flux-induced $W$ and its stringy interpretation in the different duality frames.

The orbifold involution forces the six-dimensional internal space to be factorised into three 2 -tori as shown in figure 1 . Now we will summarise the correspondence between generalised isotropic fluxes and superpotential couplings appearing in the $\mathcal{N}=1$ effective description. The generalised flux-induced superpotential can be written as

$$
W=\left(P_{F}+P_{H} S\right)+3 T\left(P_{Q}+P_{P} S\right)+3 T^{2}\left(P_{Q^{\prime}}+P_{P^{\prime}} S\right)+T^{3}\left(P_{F^{\prime}}+P_{H^{\prime}} S\right),
$$




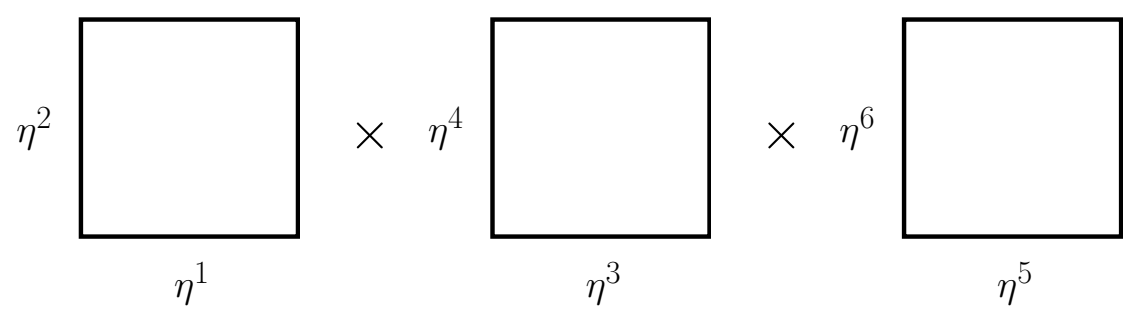

Figure 1: $T^{6}=T_{1}^{2} \times T_{2}^{2} \times T_{3}^{2}$ torus factorisation and the coordinate basis. We will use indices $a, b, c$ for horizontal "-" directions $1,3,5$ and indices $i, j, k$ for vertical "|" directions $2,4,6$ in the 2 -tori $T_{i}$ with $i=1,2,3$.

where ${ }^{2}$

$$
\begin{aligned}
& P_{F}=a_{0}-3 a_{1} U+3 a_{2} U^{2}-a_{3} U^{3} \\
& P_{Q}=c_{0}+c_{1} U-c_{2} U^{2}-c_{3} U^{3}
\end{aligned} \quad, \quad \begin{aligned}
& P_{H}=b_{0}-3 b_{1} U+3 b_{2} U^{2}-b_{3} U^{3}, \\
& P_{P}=d_{0}+d_{1} U-d_{2} U^{2}-d_{3} U^{3},
\end{aligned},
$$

and

$$
\begin{aligned}
& P_{F^{\prime}}=a_{3}^{\prime}+3 a_{2}^{\prime} U+3 a_{1}^{\prime} U^{2}+a_{0}^{\prime} U^{3} \quad, \quad P_{H^{\prime}}=b_{3}^{\prime}+3 b_{2}^{\prime} U+3 b_{1}^{\prime} U^{2}+b_{0}^{\prime} U^{3}, \\
& P_{Q^{\prime}}=-c_{3}^{\prime}+c_{2}^{\prime} U+c_{1}^{\prime} U^{2}-c_{0}^{\prime} U^{3} \quad, \quad P_{P^{\prime}}=-d_{3}^{\prime}+d_{2}^{\prime} U+d_{1}^{\prime} U^{2}-d_{0}^{\prime} U^{3} \text {. }
\end{aligned}
$$

The first half of the couplings represent fluxes which admit a locally geometric interpretation in type IIB (unprimed fluxes), whereas the remaining ones (primed fluxes) represent additional generalised fluxes which do not even admit a locally geometric description. These were first formally introduced in ref. [9] as dual counterparts of the unprimed fluxes.

For our present purposes we focus on superpotentials induced by generalised fluxes in type IIB with O3 and O7-planes which describe a locally geometric string background. Such a form of the superpotential turns out to be given by

$$
W^{\text {(loc. geom. })}=\left(P_{F}+P_{H} S\right)+3 T\left(P_{Q}+P_{P} S\right)
$$

where each polynomial $P_{F}, P_{H}, P_{Q}$ and $P_{P}$ has degree three with respect to $U$. Their explicit form and their interpretation in terms of the IIB fluxes are respectively given in (2.9) and table 1.

In this paper we will be interested in constraining the stability of solutions of these isotropic $S T U$-models originating from (generalised) string compactifications. The first relevant constraint comes from the analysis of the supersymmetry breaking sector and its

\footnotetext{
${ }^{2}$ Please note that, in principle, the truncation to the isotropic sector gives rise to $32+8=40$ fluxes, where all the fluxes transforming in the mixed symmetry representations of GL(6) (i.e. $Q, P$ and their primed counterparts in type IIB) have in fact two fluxes $\left(c_{1}, \tilde{c}_{1}\right)$ etc. giving rise to one single coupling $\left(2 c_{1}-\tilde{c}_{1}\right)$ etc., so without loss of generality, we set throughout the text $\tilde{c}_{1}=c_{1}$ etc..
} 


\begin{tabular}{|c|c|c|c|c|}
\hline couplings & Type IIB & Type IIA & fluxes & non-iso $d o f$ 's \\
\hline 1 & $F_{i j k}$ & $F_{a i b j c k}$ & $a_{0}$ & 1 \\
\hline$U$ & $F_{i j c}$ & $F_{a i b j}$ & $a_{1}$ & 3 \\
\hline$U^{2}$ & $F_{i b c}$ & $F_{a i}$ & $a_{2}$ & 3 \\
\hline$U^{3}$ & $F_{a b c}$ & $F_{0}$ & $a_{3}$ & 1 \\
\hline$S$ & $H_{i j k}$ & $H_{i j k}$ & $-b_{0}$ & 1 \\
\hline$S U$ & $H_{i j c}$ & $\omega_{i j}^{c}$ & $-b_{1}$ & 3 \\
\hline$S U^{2}$ & $H_{i b c}$ & $Q_{i}^{b c}$ & $-b_{2}$ & 3 \\
\hline$S U^{3}$ & $H_{a b c}$ & $R^{a b c}$ & $-b_{3}$ & 1 \\
\hline$T$ & $Q_{k}^{a b}$ & $H_{a b k}$ & $c_{0}$ & 3 \\
\hline$T U$ & $Q_{k}{ }^{a j}=Q_{k}{ }^{i b}, Q_{a}^{b c}$ & $\omega_{k a}^{j}=\omega_{b k}^{i}, \omega_{b c}^{a}$ & $c_{1}, \tilde{c}_{1}$ & 9 \\
\hline$T U^{2}$ & $Q_{c}^{i b}=Q_{c}{ }^{a j}, Q_{k}^{i j}$ & $Q_{b}{ }^{c i}=Q_{a}{ }^{j c}, Q_{k}{ }^{i j}$ & $c_{2}, \quad \tilde{c}_{2}$ & 9 \\
\hline$T U^{3}$ & $Q_{c}^{i j}$ & $R^{i j c}$ & $c_{3}$ & 3 \\
\hline$S T$ & $P_{k}^{a b}$ & & $-d_{0}$ & 3 \\
\hline$S T U$ & $P_{k}^{a j}=P_{k}^{i b}, P_{a}^{b c}$ & & $-d_{1},-\tilde{d}_{1}$ & 9 \\
\hline$S T U^{2}$ & $P_{c}^{i b}=P_{c}^{a j}, P_{k}^{i j}$ & & $-d_{2},-\tilde{d}_{2}$ & 9 \\
\hline$S T U^{3}$ & $P_{c}^{i j}$ & & $-d_{3}$ & 3 \\
\hline
\end{tabular}

Table 1: Mapping between unprimed fluxes and couplings in the superpotential both in type IIB with $O 3$ and $O 7$ and in type IIA with O6. The six internal directions depicted in figure 1 are split into " - " labelled by $i=1,3,5$ and "|" labelled by $a=2,4,6$. Note that the empty boxes in type IIA are related to the presence of dual fluxes analogous to the 'primed' notation in type IIB. The last column refers to the number of independent superpotential couplings the corresponding fluxes give rise to once the non-isotropic moduli are opened up.

universal features which allow one to constrain metastable de Sitter vacua [25] and inflation $[26,27]$. The averaged projection of the scalar mass matrix along the supersymmetry breaking directions results in the following sGoldstino bound for the $\eta$ parameter

$$
\eta \equiv \operatorname{Min}\left\{\text { Eigenvalues }\left(\left(m^{2}\right)^{I}{ }_{J}\right)\right\} \leq \frac{2}{3 \gamma}-\frac{1+\gamma}{\gamma} \tilde{\mathcal{R}}
$$

where $\gamma \equiv \frac{V}{3 e^{K}|W|^{2}}$ and $\tilde{\mathcal{R}}$ denotes the sectional curvature, i.e. the projection of the Riemann tensor along the sGoldstino. 


\begin{tabular}{|c|c|c|c|c|}
\hline couplings & Type IIB & Type IIA & fluxes & non-iso dof's \\
\hline$T^{3} U^{3}$ & $F^{\prime i j k}$ & & $a_{0}^{\prime}$ & 1 \\
\hline$T^{3} U^{2}$ & $F^{\prime i j c}$ & & $a_{1}^{\prime}$ & 3 \\
\hline$T^{3} U$ & $F^{\prime i b c}$ & & $a_{2}^{\prime}$ & 3 \\
\hline$T^{3}$ & $F^{\prime a b c}$ & & $a_{3}^{\prime}$ & 1 \\
\hline$S T^{3} U^{3}$ & $H^{\prime i j k}$ & & $-b_{0}^{\prime}$ & 1 \\
\hline$S T^{3} U^{2}$ & $H^{\prime i j c}$ & & $-b_{1}^{\prime}$ & 3 \\
\hline$S T^{3} U$ & $H^{\prime i b c}$ & & $-b_{2}^{\prime}$ & 3 \\
\hline$S T^{3}$ & $H^{\prime a b c}$ & & $-b_{3}^{\prime}$ & 1 \\
\hline$T^{2} U^{3}$ & $Q_{a b}^{\prime}{ }^{k}$ & & $c_{0}^{\prime}$ & 3 \\
\hline$T^{2} U^{2}$ & $Q_{a j}^{\prime}{ }^{k}=Q_{i b}^{\prime k}, Q_{b c}^{\prime a}$ & & $c_{1}^{\prime}, \tilde{c}_{1}^{\prime}$ & 9 \\
\hline$T^{2} U$ & $Q_{i b}^{\prime c}=Q_{a j}^{\prime c}, Q_{i j}^{\prime}{ }^{k}$ & & $c_{2}^{\prime}, \tilde{c}_{2}^{\prime}$ & 9 \\
\hline$T^{2}$ & $Q_{i j}^{\prime}{ }^{c}$ & & $c_{3}^{\prime}$ & 3 \\
\hline$S T^{2} U^{3}$ & $P_{a b}^{\prime}{ }^{k}$ & & $-d_{0}^{\prime}$ & 3 \\
\hline$S T^{2} U^{2}$ & $P_{a j}^{\prime}{ }^{k}=P_{i b}^{\prime k}, P_{b c}^{\prime a}$ & & $-d_{1}^{\prime},-\tilde{d}_{1}^{\prime}$ & 9 \\
\hline$S T^{2} U$ & $P_{i b}^{\prime c}=P_{a j}^{\prime c}, P_{i j}^{\prime k}$ & & $-d_{2}^{\prime},-\tilde{d}_{2}^{\prime}$ & 9 \\
\hline$S T^{2}$ & $P_{i j}^{\prime}{ }^{c}$ & & $-d_{3}^{\prime}$ & 3 \\
\hline
\end{tabular}

Table 2: Mapping between primed fluxes and couplings in the superpotential. The conventions are the as in table 1 and again, just as there, the empty column should be filled in with extra dual fluxes.

In the present supergravity setup we can explicitely evaluate the range of variation of the sectional curvature term in $(2.12) . \tilde{\mathcal{R}}$ can be written as $2 / n_{\text {eff }}$, where

$$
n_{\mathrm{eff}}=\frac{\left(G_{\alpha} G^{\alpha}\right)^{2}}{\left(G_{S} G^{S}\right)^{2}+\frac{1}{3}\left(G_{T} G^{T}\right)^{2}+\frac{1}{3}\left(G_{U} G^{U}\right)^{2}},
$$

where $G$ denotes the Kähler-invariant combination [28] $K+\log |W|^{2}$ and its first derivative $G_{\alpha}$ is normalised such that $G_{\alpha} G^{\alpha}=3(1+\gamma)$. $n_{\text {eff }}$ gives a notion of how many fields effectively participate in the supersymmetry breaking phenomenon. Such a quantity turns out to be a real number between 1 and 7, these two extremal cases corresponding, respectively, to the limits in which either $G_{\alpha}$ only points in the $S$ direction or $G_{\alpha} \sim(1,3,3)^{3}$.

\footnotetext{
${ }^{3}$ The $T$ and $U$ components are equal to 3 instead of 1 because this model originates from the $\mathrm{SL}(2)^{7}$ theory
} 
The sGoldstino bound in (2.12) implies in this case that, whenever $V>0$,

$$
n_{\mathrm{eff}} \geq 3(1+\gamma),
$$

which roughly tells us that stable dS favours homogeneous supersymmetry breaking rather than the other extremal situation in which it takes places mostly in one complex direction.

\subsection{A new systematic prescription}

Now we will present a general method for symplifying and subsequently solving the field equations in the origin of moduli space. If the scalar manifold is homogenous and the superpotential couplings constitute a closed set under non-compact duality transformations ${ }^{4}$, restricting the search for solutions to the origin of moduli space does not imply any loss of generality. This argument was first employed in ref. [19] in the context of $\mathcal{N}=4$ and $\mathcal{N}=1$ supergravity. The advantage of this is that the equations of motion for the scalar fields turn into a system of quadratic conditions for the fluxes.

Our prescription relies on a linear change of variables which introduces a number $N$ of supersymmetry breaking parameters, $N$ being the number of real fields in the model. The "broken" supersymmetry equations in the origin $\Phi_{0}$ for the complex moduli $\left\{\Phi^{\alpha}\right\}_{\alpha=1, \ldots, N / 2}$ read

$$
\left.D_{\alpha} W\right|_{\Phi_{0}}=A_{\alpha}+i B_{\alpha},
$$

where $A_{\alpha}$ and $B_{\alpha}$ denote $N$ real constants and the lhs of (2.15) is a linear expression in the $M$ fluxes. This implies that, whenever $M>N$, the above equations can always be solved in terms of $N$ fluxes as a linear function of the supersymmetry breaking parameters.

By plugging the expression of the solution of (2.15) into the field equations in the origin, one obtains a new system of equations in which the new unknowns are the $N$ supersymmetry breaking parameters and the remaining $(M-N)$ fluxes. The peculiar feature of this new system lies in the fact that the extra fluxes will now appear only linearly in the field equations, the only unknows which still appear quadratically being $A_{\alpha}$ and $B_{\alpha}$. If one arranges the supersymmetry breaking parameters into a real vector $X_{I}$ with $I=1, \ldots, N$ and denotes the fluxes by $\left\{F^{A}\right\}_{A=1, \ldots, M}$, the field equations will take the form

$$
h^{I J} X_{I} X_{J}+g_{A}^{I} X_{I} F^{A}=0,
$$

in the isotropic limit, where the triplets of complex moduli $T_{i}$ and $U_{i}$ collapse into $T$ and $U$ respectively.

${ }^{4}$ Please note that the homogeneity condition is satisfied in supergravity theories arising from $\mathbb{Z}_{2} \times \mathbb{Z}_{2}$ orbifold compactifications, both in the isotropic and in the non-isotropic case. As for the second assumption, it turns out to be true in the two examples which are relevant here, i.e. type IIA with geometric fluxes and type IIB with generalised fluxes. 
where $h$ and $g$ denote some real constants. The reason why this always has to be the case is that the supersymmetry equations should imply the field equations. Hence, whenever the rhs of (2.15) vanishes there cannot be any quadratic conditions left to be imposed on the remaining fluxes.

This makes it particularly simple to solve the field equations in the origin in the case in which the extra fluxes remaining after the introduction of the supersymmetry breaking parameters are at least $N$ (this requires $M \stackrel{!}{\geq} 2 N$ ). This sequence can be sketchily represented as

$$
\left(\begin{array}{c}
F^{1} \\
\vdots \\
F^{M}
\end{array}\right) \stackrel{(2.15)}{\rightarrow}\left(\begin{array}{c}
X_{1} \\
\vdots \\
X_{N} \\
F^{N+1} \\
\vdots \\
F^{M}
\end{array}\right) \stackrel{(2.16)}{\rightarrow}\left(\begin{array}{c}
F^{1} \\
\vdots \\
F^{2 N}
\end{array}\right)=\left(\begin{array}{c}
F^{1} \\
\vdots \\
F^{2 N}
\end{array}\right)\left(X_{1}, \ldots, X_{N}, F^{2 N+1}, \ldots, F^{M}\right)
$$

A situation which then turns out to be of special interest is that of having exactly $2 N$ fluxes turned on, so that one is able to write down the general solution to the field equations by expressing all the fluxes as a function of the supersymmetry breaking parameters. The $N$-paramater family of solutions generated in this way should provide, at least as a matter of principle, enough freedom to have control upon the eigenvalues of mass matrix for the $N$ scalars retained in the theory. Generically, this tachyon-free region in parameter space will have a non-trivial intersection with the $\mathrm{dS}$ region. This situation is conceptually depicted in figure 2 .

Our perspective points towards the possibility that, by introducing a non-isotropic setup admitting a 14-dimensional space of solutions, one might have enough freedom for giving a positive mass to all the scalars in the $\mathrm{SL}(2)^{7}$ model. At least qualitatively, increasing the number $N$ of fields should not spoil this picture. The issue that we will address in the next section will be extimating how small the region of stable $\mathrm{dS}$ is compared to the whole parameter space of solutions. This will tell us how difficult it practically becomes to scan the whole parameter space searching for stable dS vacua. This analysis, on the contrary, will certainly depend on $N$; in order to make predictions, we will now focus on the simpler $N=6$ case corresponding with the isotropic model.

\subsection{Geometric IIA backgrounds: an over-constrained example}

Let us now consider the type IIA duality frame with O6-planes. This class of compactifications fits again the framework of $\mathcal{N}=1$ effective descriptions in that there are two different 


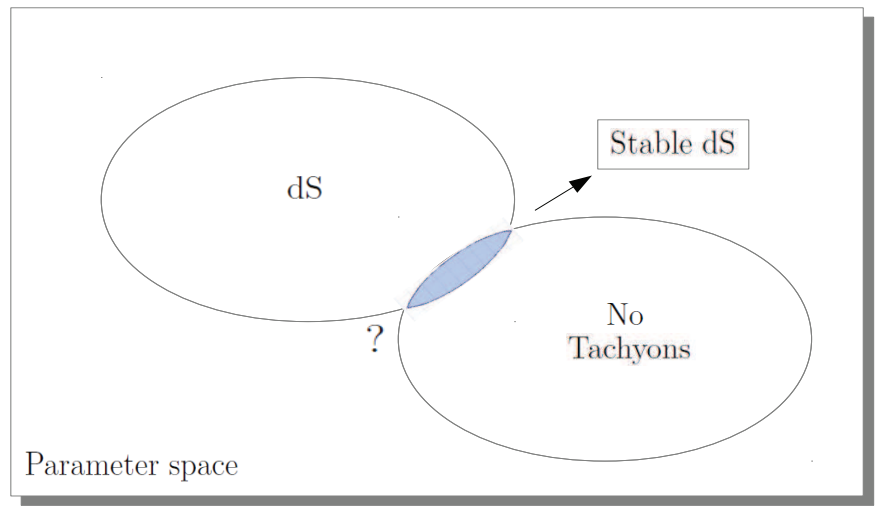

Figure 2: The $d S$ region as well as the tachyon-free region are subsets of positive measure in the $N$-dimensional parameter space of solutions. Generically, they will overlap in a region that may be small but still is expected to be of positive measure.

kinds of allowed D6-branes, i.e. those ones parallel to the O-planes which do not further break supersymmetry and those ones orthogonal to them which break supersymmetry down to $\mathcal{N}=1$. In this context, in the isotropic limit, one can have the four different $\mathrm{R}$ - $\mathrm{R}$ gauge fluxes $\left(\left\{F_{p}\right\}_{p=0,2,4,6}\right)$, two independent components of $H$ flux, and three components of metric flux $\omega$, of which only two generate independent superpotential couplings (see footnote 2). The corresponding superpotential reads

$$
W^{\text {(geom. IIA) }}=\left(a_{0}-3 a_{1} U+3 a_{2} U^{2}-a_{3} U^{3}\right)+\left(b_{0}-3 b_{1} U\right) S+3 T\left(c_{0}+c_{1} U\right),
$$

where the type IIA interpretation of the above couplings can be found in table 1. In this case, setting $\tilde{c}_{1}=c_{1}$ as discussed in footnote 2 has a special geometric interpretation, because it represents the correct way of solving the constraints $\omega^{2}=0$ and $\omega H=0$ which ensure the closure of the exterior derivative defined by the corresponding NS-NS background.

We choose the origin of moduli space to be

$$
S_{0}=T_{0}=U_{0}=i
$$

By applying the counting introduced in section 2.2, one immediately realises that the amount $M=8$ of fluxes is not large enough for following the above prescription for solving the equations of motion in the origin. After introducing the six supersymmetry breaking parameters 
by solving the (2.15), one finds

$$
\begin{aligned}
& a_{2}=\frac{1}{9}\left(-B_{1}-B_{2}+2 B_{3}\right) \\
& a_{3}=\frac{1}{9}\left(-3 A_{1}-3 A_{2}+2 A_{3}\right), \\
& b_{0}=\frac{1}{9}\left(-6 A_{1}+3 A_{2}+A_{3}\right) \\
& b_{1}=\frac{1}{9}\left(2 B_{1}-B_{2}-B_{3}\right) \\
& c_{0}=-\frac{1}{9}\left(3 A_{1}+A_{3}\right) \\
& c_{1}=-\frac{1}{3}\left(B_{1}+B_{3}\right)
\end{aligned}
$$

After solving two axionic equations of motion by $a_{0}=a_{1}=0$, one is left with the following field equations

$$
\begin{array}{r}
-3 A_{1}^{2}+2 A_{1} A_{3}-2 A_{2} A_{3}+A_{3}^{2}-3 B_{1}^{2}-2 B_{1} B_{3}-2 B_{2} B_{3}+B_{3}^{2}=0, \\
9 A_{1}^{2}-6 A_{1}\left(A_{2}+A_{3}\right)-2 A_{2} A_{3}+3 A_{3}^{2}+9 B_{1}^{2}-6 B_{1} B_{2}-6 B_{1} B_{3}-6 B_{2} B_{3}+3 B_{3}^{2}=0, \\
9 A_{1}^{2}-9 A_{1} A_{2}+3 A_{1} A_{3}+3 A_{2} A_{3}+3 B_{1}^{2}-3 B_{1} B_{2}-B_{1} B_{3}-B_{2} B_{3}-4 B_{3}^{2}=0 \\
6 A_{1} B_{1}-3 A_{1} B_{2}+3 A_{1} B_{3}-3 A_{2} B_{1}+3 A_{2} B_{3}+A_{3} B_{1}+A_{3} B_{2}=0 .
\end{array}
$$

Based on a technique from algebraic geometry called prime ideal decomposition and its implementation in the software Singular [29], one can decompose the system (2.20) into its prime ideals which can be solved separately and give rise to disconnected branches of the general solution. Only one of these branches happens to contain dS extrema and these organise themselves into a one-parameter family of solutions. This dS line is plotted in figure 3 together with the value of the $\eta$ parameter as a function of the scan parameter, which shows the presence of tachyons at every point. On the other hand, this over-constrained situation already suggests that one might not be able to tune the masses of the six isotropic real scalars and get stable dS points. Further in figure 4 we show the normalised energy $\gamma$ and its corresponding critical value saturating the sGoldstino bound in (2.14). This analysis manifests that the averaged sGoldstino (over real and imaginary parts) is never tachyonic all along the dS, with $\gamma$ that stays orders of magnitude away from the critical value and only approaches it in the singular regime in which $\delta$ goes to infinity ${ }^{5}$.

\footnotetext{
${ }^{5}$ For further discussion on the presence of tachyons in these models, and the $\delta$ limit in particular, see ref. [16].
} 

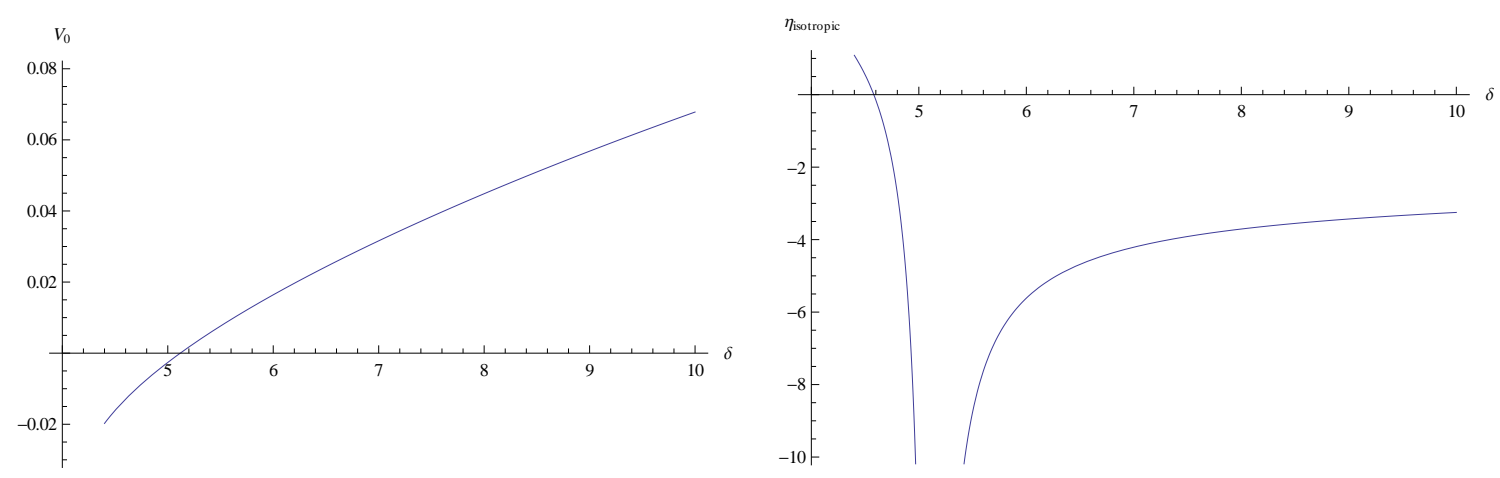

Figure 3: Left: the one-parameter branch of $d S$ solutions of geometric type IIA isotropic flux compactifications. The value of the energy $V_{0}$ is given as a function of the scan parameter $\delta=\exp \left(A_{1}\right)$. The curve crosses the Minkowski line at $\delta \sim 5.127$.

Right: the value of the $\eta$ parameter within the isotropic sector as a function of the scan parameter $\delta$ already reveals the presence of tachyons all along the $d S$ branch.

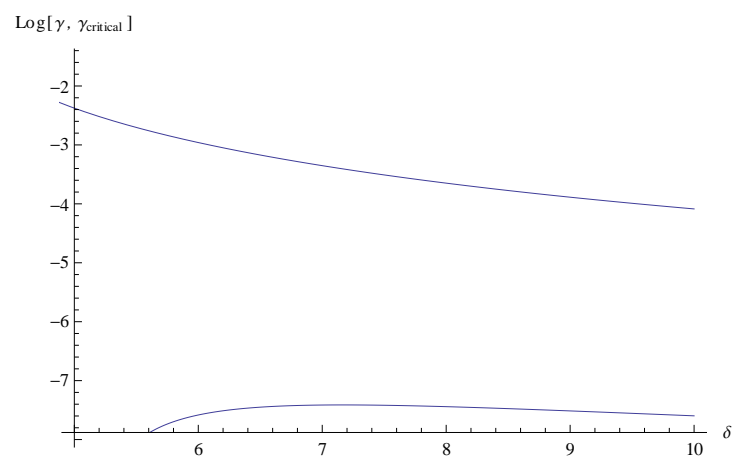

Figure 4: Comparison between the value of $\gamma$ along the $d S$ line and $\gamma_{\text {critical }}=\frac{n_{\text {eff }}}{3}-1$, i.e. the maximal value of $\gamma$ for which the averaged sGoldstino mass is not negative.

\section{Towards stable dS solutions}

In the previous section we have reviewed the case of geometric type IIA compactifications as an over-constrained example to which one can think of applying the method presented in section 2.2. Now we would like to move to the most interesting situation in which $M$ exactly equals $2 N$. In our isotropic setup $(N=6)$ this implies the necessity of having 12 fluxes. By taking a look at tables 1 and 2, one realises immediately that this requires including non-geometric fluxes both in type IIA and in type IIB. 


\subsection{A type IIB setup with generalised fluxes}

The setup we focus on is the IIB duality frame with O3 and O7-planes with generalised fluxes defining a locally geometric background, i.e. $F_{3}, H, Q$ and $P$ fluxes. The induced superpotential is the one given in (2.11). The aim of this section will be solving the equations of motion in the origin (2.18) for the isotropic scalars in order to find the most general set of extrema in this case and study the stability features thereof.

We will analyse here the special sub-case of a generalised type IIB background with only $F_{3}, H$ and $Q$ fluxes and vanishing $P$ flux. The general superpotential in this case is given in (A.1). This choice is motivated by the fact that, in this non-geometric setup, a dS critical point was found in ref. [17] with the interesting property of being stable with respect to the six isotropic scalars. This supergravity solution, though, reveals the presence of tachyons in the non-isotropic directions of the scalar manifold. We will now solve the equations of motion for the scalars in this setup and show that the solutions span a parameter space of dimension six, in which the stable dS point of [17] sits.

In general, given such a 6-dimensional parameter space, one can only hope to have control on the eigenvalues of the mass matrix in the six isotropic directions and there is no freedom left to stabilise the extra 8 non-isotropic scalars which could then remain tachyonic.

The method for finding the general exact solution to the field equations in this nongeometric type IIB setup is presented in appendix A.

\subsection{Statistical scan and tests of RMT}

In the previous section we have motivated our interest in the study of non-geometric type IIB compactifications with $F_{3}, H$ and $Q$ fluxes. This seems to be the minimal setup for producing stable dS solutions at least for what concerns the mass spectrum within the isotropic sector. At least qualitatively we have argued that this situation in which $N=6$ fields are retained can be regarded as a toy example for extrapolating some universal features of the probability distribution of stable critical points for potentials with $N$ fields in general.

Analysing the probability distribution of stationary points of different types (i.e. minima, maxima or saddle points) for classes of scalar potentials is equivalent to studying the distribution of the eigenvalues of its Hessian matrix evaluated at those given points. In the case in which one assumes the scalar potential to be a random function, its Hessian matrix will be a symmetric matrix with random entries.

The distribution of the eigenvalues of a random matrix is the subject studied by Random Matrix Theory (RMT). This framework has been used in refs [30-33] in order to predict the heavy suppression of stable dS vacua in string-inspired supergravity models with $N$ large 
enough.

Let us now go back to our general solutions presented in section 3 and appendix A. Even though this set of exact solutions is obtained analytically, one immediately realises that scanning a parameter space of dimension six in order to find stable vacua requires a different approach, since the analytical beahviour of the eigenvalues of the mass matrix as a function of these parameters is out of reach.

What we did was generating a statistically significant sample of random values for the six supersymmetry breaking parameters $\left\{A_{\alpha}, B_{\alpha}\right\}_{\alpha=S, T, U}$, thus yielding an equally big sample of random points in the space of solutions. In figure 5 we show the resulting probability distribution of tachyon-free critical points as a function of the scanning parameter ${ }^{6} \tilde{\gamma}$ rep- $^{-}$ resenting the ratio between the positive contribution to the cosmological constant and the AdS scale.

This result shows the presence of a peak of tachyon-free points in the AdS region with small $\tilde{\gamma}$ but away from the supersymmetric point at $\tilde{\gamma}=0$. This could be explained by observing that the mass spectrum in the supersymmetric case is completely determined by the masses $m_{\alpha \beta}$ of the chiral fermionic superpartners of the scalars. When these are massless, the corresponding partener scalars sit at the conformal value $m^{2}=-\frac{2}{3}$. Non-zero fermionic masses will produce linear corrections to the off-diagonal elements of the scalar mass matrix and quadratic ones to its diagonal entries. By studying in detail the sign of the eigenvalues, one finds the presence of at least one tachyon (though obviously still above the BF bound) as a generic feature, whereas introducing supersymmetry breaking makes it slightly easier to arrange for all positive eigenvalues.

Furthermore, there appears a second sharp peak in figure 5 in the AdS region but with values of $\tilde{\gamma}$ close to 1 . We do not have a clear explanation for this at the moment, though it seems to indicate that there might be a natural way of saturating the sGoldstino bound for the $\eta$ parameter in the AdS region for a certain value of $\gamma$.

Finally, we observe a tiny tail of stable dS points with the very specific feature of having $\tilde{\gamma}$ slightly above 1 . This seems to be in line with the results in refs [34-36] where the relation between flux-induced potentials and RMT predictions is discussed, the final outcome being that string compactifications strongly suppress stable dS vacua with large values of the cosmological constant.

In what follows, we would also like to make a comparison between our present analysis and the RMT-based results. It is important to keep in mind that our supergravity models describing a background with generalised fluxes only allows for a small number of random

\footnotetext{
${ }^{6}$ Please not that $\tilde{\gamma}=\gamma+1, \gamma$ being the normalised energy parameter introduced in the context of the sGoldstino analysis.
} 
parameters that equals the number of diagonal entries of the mass matrix, whereas all the remaining off-diagonal ones are determined in terms of these by the equations of motion. In other words, while the RMT is dealing with a more or less arbitrary random superpotential, we are considering a constrained string inspired subset.

We do not expect the RMT description to be applicable when the supersymmetry breaking scale and the AdS scale are of comparable size $(\tilde{\gamma} \gtrsim 1)$. However, it is interesting to make a comparison in the far-right region where $\tilde{\gamma}$ is significantly larger than the Minkowski value of 1 . Using the estimate $[32,37]$

$$
\mathcal{P}=\exp \left[-\frac{\log 3}{4} N^{2}+\frac{\log (2 \sqrt{3}-3)}{2} N-\frac{\log N}{24}-0.0172\right],
$$

one finds an estimated fraction $4.36 \times 10^{-6}$ of stable dS for $N=6$. This would predict roughly 25 to 30 stable solutions out of the total of $\sim 6.3 \times 10^{6}$ dS solutions with $\tilde{\gamma}>1.5$ in our scan. In contrast, we were unable to find a single example of stable dS in this region. This significant deviation from the RMT prediction confirms our expectation that there is actually no regime in which our $\mathcal{N}=1$ supergravity potentials, inspired by non-geometric string backgrounds, fit an RMT approximation.

If one wants to reproduce the RMT predictions for stable dS vacua in the high $\tilde{\gamma}$ region, it is clear that one needs to include many more random parameters. The stringy origin of these remains, however, an open issue.

As a motivation towards further work in this direction, we would like to give a first look at the $N=14$ case corresponding to studying the full $\mathrm{SL}(2)^{7}$ model relaxing isotropy. The analgous setup allowing for enough freedom in parameter space will now have 28 fluxes. By taking a look at the last column of table 1, one observes that also in this non-isotropic situation one needs to consider non-geometric backgrounds in any duality frame. As an example of this, we chose a type IIB background with the most general gauge fluxes $\left(F_{3}\right.$ and $H$ ) plus those $Q$ flux components appearing in superpotential terms with up to linear dependence on $U_{i}$ (The first two types of $Q$ flux in table 1). These fluxes turn out to be exactly 28 in total.

In figure 6 we give an idea of the results for the random scan in this case. These preliminary results were obtained by means of a limited sample of data and we leave a detailed analysis for future purposes. Such a study will certainly be needed in order to investigate the presence of fully stable dS vacua, if any. However, the present diagram of the probability distribution of tachyon-free points w.r.t. all real moduli roughly shows some common features with the $N=6$ case in the AdS region. 


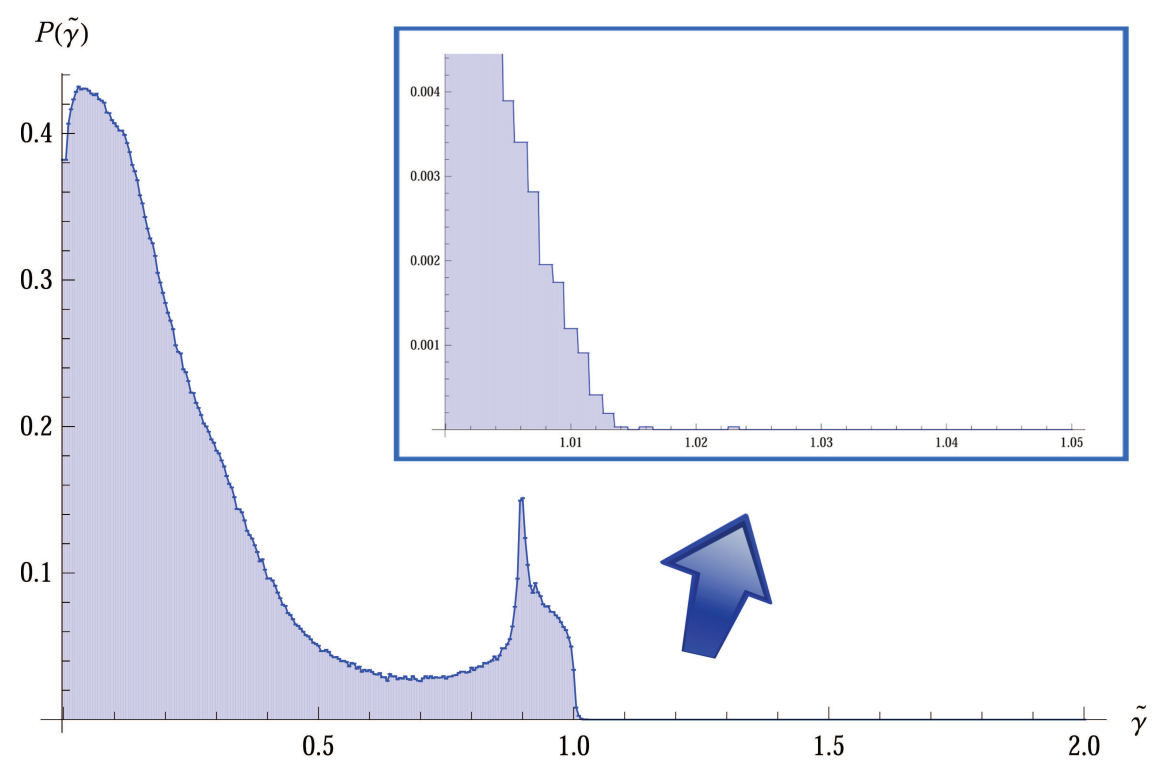

Figure 5: Distribution of the fraction of tachyon-free critical points of the isotropic scalar potential with $N=6$ fields as a function of the uplift parameter $\tilde{\gamma} \equiv \frac{|D W|^{2}}{3|W|^{2}}$. Data produced from the analysis of $10^{7}$ random points. The zoomed diagram in the upper-right corner shows the presence of a stable $d S$ tail with $1<\tilde{\gamma} \lesssim 1.02$. This tail was analysed using a sample bigger by a factor of 10 .

\subsection{Analysis of the stable dS region}

Now we would like to carry out a complementary analysis w.r.t. the statistical scan presented in the previous section. The aim is that of charting the region of stable dS inside parameter space in the IIB case previously introduced and studied. In this way, one can think of explicitely construct the concrete form of the conceptual picture given in figure 2 which applies in this case.

Since the parameter space of solutions has dimension six, we studied different twodimensional slices thereof. We have fixed four out of six supersymmetry breaking parameters to the values corresponding to the stable dS solution in ref. [17] and moved about in the remaining two-dimensional slice. We have constructed the level curves of the scalar potential around such a point and the level curves of the minimal eigenvalue of the mass matrix. Finally, we have studied the overlap between the dS region and the tachyon-free region thus locating stable dS points in parameter space. The results of such an analysis are collected in figures 7 and 8, which represent the $\left(\Re\left(D_{S} W\right), \Im\left(D_{S} W\right)\right)$-plane and the $\left(\Re\left(D_{S} W\right), \Re\left(D_{T} W\right)\right)$-plane respectively. 


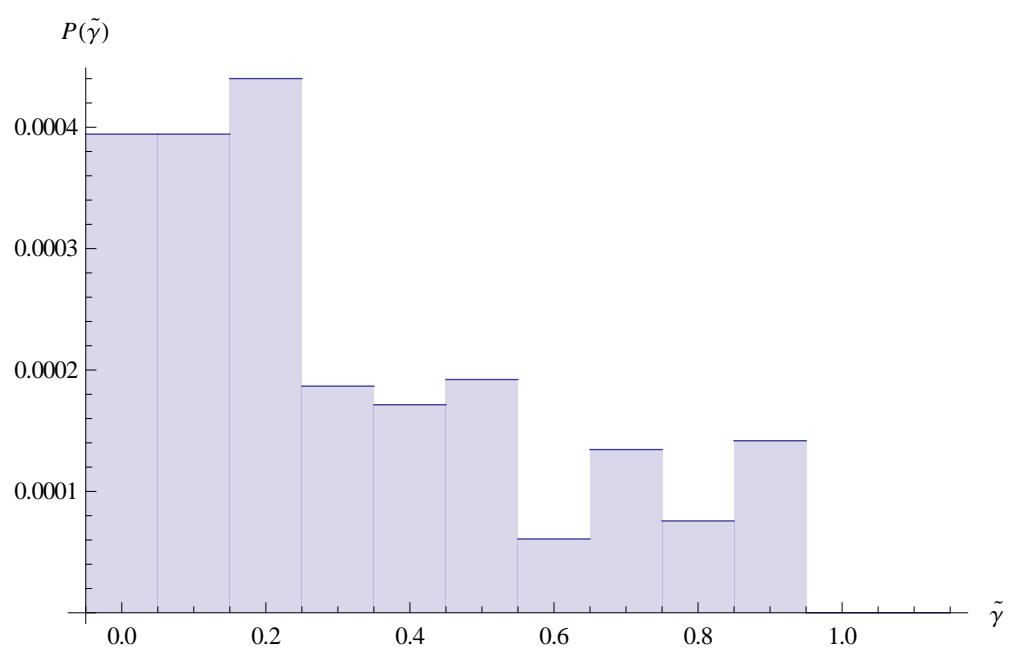

Figure 6: Distribution of the fraction of tachyon-free critical points of the full scalar potential with $N=14$ fields as a function of the uplift parameter $\tilde{\gamma} \equiv \frac{|D W|^{2}}{3|W|^{2}}$. Data produced from the analysis of $5 \times 10^{5}$ random points. Although the statistical significance is not sufficient for detecting any stable Minkowski or $d S$ tail, the general features in the AdS region are compatible with the results obtained for $N=6$.
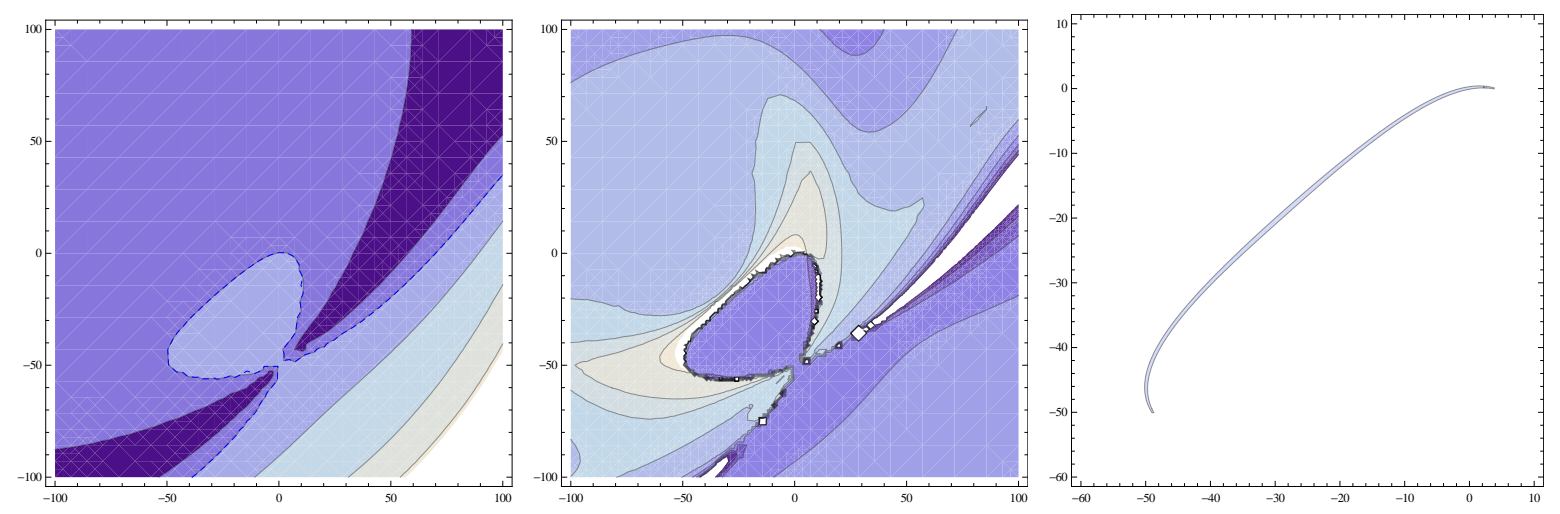

Figure 7: The parameter space of solutions projected on the $\left(A_{1}, B_{1}\right)$ plane. Left: Level curves of the cosmological constant; the lower-right corner filled with lighter colours and delimited by the Minkowski (blue dashed) line corresponds to the $d S$ region. Middle: Level curves of the $\eta$ parameter; the tachyon-free region corresponds to the upper-left part filled with lighter colours. Right: The tiny region of overlap in parameter space corresponding with stable dS is zoomed in here.

The striking peculiarity of the $\mathrm{dS}$ region and the tachyon-free region is that they happen to be almost each other's negative and this also holds for all the other two-dimensional slices that are not given here. This always makes the corresponding overlap region look like a very 

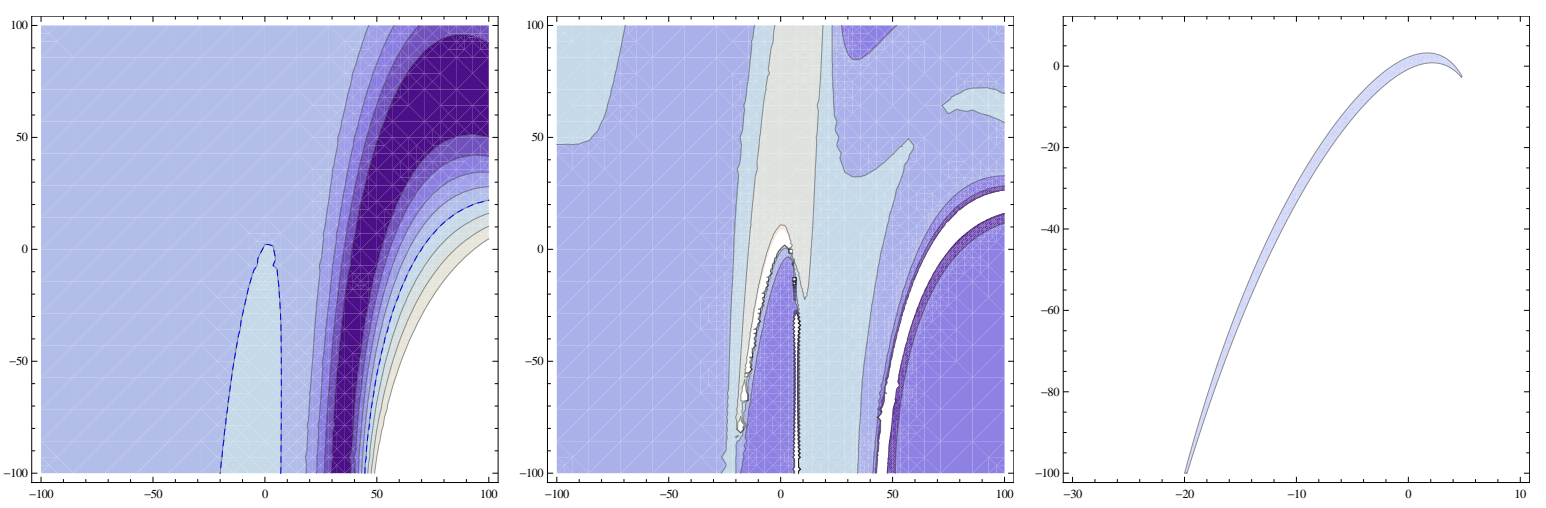

Figure 8: The parameter space of solutions projected on the $\left(A_{1}, A_{2}\right)$ plane. Left: Level curves of the cosmological constant; the $d S$ regions are the ones filled with lighter colours below by the Minkowski (blue dashed) lines. Middle: Level curves of the $\eta$ parameter; the tachyon-free regions are filled with lighter colours. Right: The tiny region of overlap in parameter space corresponding with stable $d S$ is zoomed in here.

narrow band. By moving away from the stable dS point in which the plots are centered, the stable dS band tends to persist. This may be regarded as an indication for the stable dS region to have the shape of a thick sheet in six dimensions, in which one of the dimensions is much narrower than the other ones. Studying the topology of such a stable dS region and in particular, as a first thing, understanding whether it consists of or more connected components, requires some further detailed investigation that we leave for future work.

Furthermore, this complementary analysis corroborates what the random analysis was already suggesting, i.e. that the very tiny stable dS regions always occurs very close a Minkowski critical point and hence with values of $\tilde{\gamma}$ slightly above 1 .

\section{Conclusions}

In this paper we have offered a framework for systematically analysing the space of solutions of type II string compactifications compatible with $\mathcal{N}=1$ supersymmetry. The supergravity effective description realises string dualities as global symmetries, and therefore it comprises different descriptions in different duality frames in a single universal formulation.

Supersymmetry breaking parameters allow one to predict the dimensionality of the space of solutions by means of a very simple counting argument. In this context we were able to revisit the case of geometric type IIA compactifications. We find that the space of solutions does not seem to contain enough degrees of freedom for getting rid of the tachyons even within the isotropic sector. 
Subsequently, we applied this novel prescription to investigate less constrained situations such as isotropic IIB compactifications with generalised fluxes. A statistical scan as well as a charting of two-dimensional slices of the parameter space reveal the presence of thin sheets of stable dS regions with small cosmological constant (compared to the AdS scale). Generically, since our parameter space is just big enough for controlling the eigenvalues of the mass matrix in the isotropic sector, we expect that the remaining non-isotropic sector will contain tachyons. This we have checked in some explicit cases, even though we have not performed a full analysis.

However, by considering non-isotropic setups with a larger parameter space, our general expectation is that there will exist a non-empty stable dS region. This region will probably be considerably thinner than its isotropic counterpart. Indeed, a first random scan of the nonisotropic case (although not with enough statistical significance), has exhibited no examples of tachyon-free dS critical points. This means that the issue of the existence of a fully stable $\mathrm{dS}$ vacuum, w.r.t. to all fourteen non-isotropic moduli, still remains to be addressed. We hope to come back to this point in the future.

Our work shows that stable de Sitter vacua are much less frequent than one naively would expect. In particular, we have observed the necessity to add at least some flux that is non-geometric from both the type IIA and the type IIB point of view. Furthermore, our analysis hints at tantalizing conspiracies, which suggest that dS vacua, if at all present, have unnaturally small values (compared to the AdS scale). It would be interesting to analytically understand the reason for these conspiracies, and to investigate their possible phenomenological consequences.

\section{Acknowledgments}

We would like to thank Johan Blåbäck, Adolfo Guarino, Joseph Minahan, Oscar Varela for stimulating discussions. We are also especially greatful to Gary Shiu, Thomas Van Riet and Timm Wrase for valuable comments on a draft version of this manuscript. The work of the authors is supported by the Swedish Research Council (VR), and the Göran Gustafsson Foundation. 


\section{A All the solutions of type IIB with $F, H$ and $Q$}

The general form of the superpotential is obtained by taking (2.8) and switching of all the couplings but those ones in $P_{F}, P_{H}$ and $P_{Q}$. This yields

$$
\begin{aligned}
W^{(P=0)} & =a_{0}-3 a_{1} U+3 a_{2} U^{2}-a_{3} U^{3}+\left(b_{0}-3 b_{1} U+3 b_{2} U^{2}-b_{3} U^{3}\right) S+ \\
& +3 T\left(c_{0}+c_{1} U-c_{2} U^{2}-c_{3} U^{3}\right)
\end{aligned}
$$

After solving the "broken" supersymmetry conditions (2.15) in terms of $\left\{b_{2}, b_{3}, c_{0}, c_{1}, c_{2}, c_{3}\right\}$ via

$$
\begin{aligned}
& b_{2}=\frac{1}{6}\left(3 a_{1}-a_{3}+2 b_{0}+A_{1}-A_{2}\right) \\
& b_{3}=\frac{1}{2}\left(-a_{0}+3 a_{2}+6 b_{1}-B_{1}+B_{2}\right) \\
& c_{0}=\frac{1}{12}\left(9 a_{1}+a_{3}+4 b_{0}-A_{1}-A_{2}-2 A_{3}\right) \\
& c_{1}=\frac{1}{12}\left(9 a_{0}-15 a_{2}-12 b_{1}-3 B_{1}-3 B_{2}-2 B_{3}\right) \\
& c_{2}=\frac{1}{12}\left(9 a_{1}-7 a_{3}-4 b_{0}-5 A_{1}-A_{2}+2 A_{3}\right) \\
& c_{3}=\frac{1}{12}\left(-3 a_{0}-3 a_{2}+12 b_{1}-3 B_{1}+B_{2}+2 B_{3}\right)
\end{aligned}
$$

one finds

$$
\begin{aligned}
& \left(6 B_{1}+3 B_{2}-3 B_{3}\right) a_{0}+3\left(6 A_{1}+3 A_{2}-A_{3}\right) a_{1}-9\left(2 B_{1}+B_{2}-B_{3}\right) a_{2}+ \\
& -\left(6 A_{1}+3 A_{2}-A_{3}\right) a_{3}-8 A_{3} b_{0}+24 B_{3} b_{1}-6 A_{1}^{2}-A_{1}\left(3 A_{2}+A_{3}\right)-6 B_{1}^{2}+ \\
& -3 B_{1} B_{2}-3 B_{1} B_{3}-A_{2}^{2}+A_{2} A_{3}-B_{2}^{2}+3 B_{2} B_{3}+2 A_{3}^{2}+2 B_{3}^{2}=0 \\
& 3\left(2 A_{1}-A_{2}+A_{3}\right) a_{0}-3\left(6 B_{1}-3 B_{2}+B_{3}\right) a_{1}-9\left(2 A_{1}-A_{2}+A_{3}\right) a_{2}-8 B_{3} b_{0}-24 A_{3} b_{1}+ \\
& +\left(6 B_{1}-3 B_{2}+B_{3}\right) a_{3}-A_{1}\left(3 B_{2}+B_{3}\right)+3 B_{1} A_{2}+3 B_{1} A_{3}+A_{2} B_{3}-3 B_{2} A_{3}=0 \\
& 3\left(3 B_{1}+4 B_{2}+3 B_{3}\right) a_{0}+9\left(3 A_{1}+4 A_{2}+A_{3}\right) a_{1}-3\left(9 B_{1}+12 B_{2}+B_{3}\right) a_{2}+ \\
& -\left(9 A_{1}+12 A_{2}-5 A_{3}\right) a_{3}+8 A_{3} b_{0}-24 B_{3} b_{1}+9 A_{1}^{2}+A_{1}\left(-15 A_{2}+A_{3}\right)+9 B_{1}^{2}+ \\
& -15 B_{1} B_{2}+3 B_{1} B_{3}-4 A_{2}^{2}-A_{2} A_{3}-4 B_{2}^{2}-3 B_{2} B_{3}+2 A_{3}^{2}+2 B_{3}^{2}=0 \\
& -9\left(A_{1}+A_{3}\right) a_{0}+9\left(3 B_{1}+B_{3}\right) a_{1}+3\left(9 A_{1}+A_{3}\right) a_{2}-\left(9 B_{1}-5 B_{3}\right) a_{3}+ \\
& +8 B_{3} b_{0}+24 A_{3} b_{1}+A_{1}\left(3 B_{2}+B_{3}\right)-3 B_{1} A_{2}-3 B_{1} A_{3}-A_{2} B_{3}+3 B_{2} A_{3}=0 \\
& -9\left(B_{1}-B_{2}-2 B_{3}\right) a_{0}+3\left(-3 A_{1}+3 A_{2}+2 A_{3}\right) a_{1}+3\left(9 B_{1}-B_{2}-2 B_{3}\right) a_{2}+ \\
& +\left(3 A_{1}+5 A_{2}-18 A_{3}\right) a_{3}+8 A_{2} b_{0}-24 B_{2} b_{1}-3 A_{1}^{2}+A_{1}\left(-24 b_{0}+4 A_{2}\right)-9 B_{1}^{2}+ \\
& +72 B_{1} b_{1}+12 B_{1} B_{2}-A_{2}^{2}-4 A_{2} A_{3}-3 B_{2}^{2}-4 B_{2} B_{3}+4 A_{3}^{2}-4 B_{3}^{2}=0 \\
& 3\left(3 A_{1}-3 A_{2}-2 A_{3}\right) a_{0}-3\left(3 B_{1}-3 B_{2}+10 B_{3}\right) a_{1}-3\left(9 A_{1}-A_{2}+10 A_{3}\right) a_{2}+ \\
& +\left(3 B_{1}+5 B_{2}-6 B_{3}\right) a_{3}+8 B_{2} b_{0}+24 A_{2} b_{1}-A_{1}\left(-6 B_{1}+72 b_{1}+8 B_{2}\right)+ \\
& -24 B_{1} b_{0}+2 A_{2} B_{2}+4 A_{2} B_{3}-4 B_{2} A_{3}+8 A_{3} B_{3}=0 . \\
&
\end{aligned}
$$


The above set of equations can be then solved with respect to $\left\{a_{0}, a_{1}, a_{2}, a_{3}, b_{0}, b_{1}\right\}$ which

only appear linearly. This procedure combined with (A.2) gives rise to to the general solution expressing the 12 fluxes as a function of the six supersymmetry breaking parameters.

\section{References}

[1] S. B. Giddings, S. Kachru, and J. Polchinski, "Hierarchies from fluxes in string compactifications," Phys.Rev. D66 (2002) 106006, arXiv:hep-th/0105097 [hep-th].

[2] S. Kachru, M. B. Schulz, and S. Trivedi, "Moduli stabilization from fluxes in a simple IIB orientifold," JHEP 0310 (2003) 007, arXiv:hep-th/0201028 [hep-th].

[3] J.-P. Derendinger, C. Kounnas, P. M. Petropoulos, and F. Zwirner, "Superpotentials in IIA compactifications with general fluxes," Nucl.Phys. B715 (2005) 211-233, arXiv:hep-th/0411276 [hep-th].

[4] O. DeWolfe, A. Giryavets, S. Kachru, and W. Taylor, "Enumerating flux vacua with enhanced symmetries," JHEP 0502 (2005) 037, arXiv:hep-th/0411061 [hep-th].

[5] P. G. Camara, A. Font, and L. Ibanez, "Fluxes, moduli fixing and MSSM-like vacua in a simple IIA orientifold," JHEP 0509 (2005) 013, arXiv:hep-th/0506066 [hep-th].

[6] G. Villadoro and F. Zwirner, "N=1 effective potential from dual type-IIA D6/O6 orientifolds with general fluxes," JHEP 0506 (2005) 047, arXiv:hep-th/0503169 [hep-th].

[7] J.-P. Derendinger, C. Kounnas, P. Petropoulos, and F. Zwirner, "Fluxes and gaugings: N=1 effective superpotentials," Fortsch.Phys. 53 (2005) 926-935, arXiv:hep-th/0503229 [hep-th].

[8] O. DeWolfe, A. Giryavets, S. Kachru, and W. Taylor, "Type IIA moduli stabilization," JHEP 0507 (2005) 066, arXiv:hep-th/0505160 [hep-th].

[9] G. Aldazabal, P. G. Camara, A. Font, and L. Ibanez, "More dual fluxes and moduli fixing," JHEP 0605 (2006) 070, arXiv:hep-th/0602089 [hep-th].

[10] G. Aldazabal and A. Font, "A Second look at N=1 supersymmetric AdS(4) vacua of type IIA supergravity," JHEP 0802 (2008) 086, arXiv:0712.1021 [hep-th].

[11] R. Flauger, S. Paban, D. Robbins, and T. Wrase, "Searching for slow-roll moduli inflation in massive type IIA supergravity with metric fluxes," Phys.Rev. D79 (2009) 086011, arXiv:0812.3886 [hep-th]. 
[12] C. Caviezel, P. Koerber, S. Kors, D. Lust, T. Wrase, et al., "On the Cosmology of Type IIA Compactifications on SU(3)-structure Manifolds," JHEP 0904 (2009) 010, arXiv:0812.3551 [hep-th].

[13] U. H. Danielsson, P. Koerber, and T. Van Riet, "Universal de Sitter solutions at tree-level," JHEP 1005 (2010) 090, arXiv:1003.3590 [hep-th].

[14] U. H. Danielsson, S. S. Haque, G. Shiu, and T. Van Riet, "Towards Classical de Sitter Solutions in String Theory," JHEP 0909 (2009) 114, arXiv:0907.2041 [hep-th].

[15] U. H. Danielsson, S. S. Haque, P. Koerber, G. Shiu, T. Van Riet, et al., "De Sitter hunting in a classical landscape," Fortsch.Phys. 59 (2011) 897-933, arXiv:1103.4858 [hep-th].

[16] U. H. Danielsson, G. Shiu, T. Van Riet, and T. Wrase, "A note on obstinate tachyons in classical dS solutions," JHEP 1303 (2013) 138, arXiv:1212.5178 [hep-th].

[17] B. de Carlos, A. Guarino, and J. M. Moreno, "Flux moduli stabilisation, Supergravity algebras and no-go theorems," JHEP 1001 (2010) 012, arXiv:0907.5580 [hep-th].

[18] G. Dibitetto, R. Linares, and D. Roest, "Flux Compactifications, Gauge Algebras and De Sitter," Phys.Lett. B688 (2010) 96-100, arXiv:1001.3982 [hep-th].

[19] G. Dibitetto, A. Guarino, and D. Roest, "Charting the landscape of N=4 flux compactifications," JHEP 1103 (2011) 137, arXiv:1102.0239 [hep-th].

[20] B. de Carlos, A. Guarino, and J. M. Moreno, "Complete classification of Minkowski vacua in generalised flux models," JHEP 1002 (2010) 076, arXiv:0911.2876 [hep-th].

[21] J. Shelton, W. Taylor, and B. Wecht, "Nongeometric flux compactifications," JHEP 0510 (2005) 085, arXiv:hep-th/0508133 [hep-th].

[22] A. Font, L. E. Ibanez, D. Lust, and F. Quevedo, "Supersymmetry Breaking From Duality Invariant Gaugino Condensation," Phys.Lett. B245 (1990) 401-408.

[23] E. Witten, "Nonperturbative superpotentials in string theory," Nucl.Phys. B474 (1996) 343-360, arXiv:hep-th/9604030 [hep-th].

[24] A. Achucarro, B. de Carlos, J. Casas, and L. Doplicher, "De Sitter vacua from uplifting D-terms in effective supergravities from realistic strings," JHEP 0606 (2006) 014, arXiv:hep-th/0601190 [hep-th].

[25] L. Covi, M. Gomez-Reino, C. Gross, J. Louis, G. A. Palma, et al., "Constraints on modular inflation in supergravity and string theory," JHEP 0808 (2008) 055, arXiv:0805.3290 [hep-th]. 
[26] A. Borghese, D. Roest, and I. Zavala, "A Geometric bound on F-term inflation," JHEP 1209 (2012) 021, arXiv:1203.2909 [hep-th].

[27] A. Achucarro, S. Mooij, P. Ortiz, and M. Postma, "Sgoldstino inflation," JCAP 1208 (2012) 013, arXiv:1203.1907 [hep-th].

[28] E. Cremmer, B. Julia, J. Scherk, S. Ferrara, L. Girardello, et al., "Spontaneous Symmetry Breaking and Higgs Effect in Supergravity Without Cosmological Constant," Nucl.Phys. B147 (1979) 105.

[29] W. Decker, G.-M. Greuel, G. Pfister, and H. Schönemann, "Singular 3-1-2 - A computer algebra system for polynomial computations,". http://www.singular.uni-kl.de.

[30] A. Aazami and R. Easther, "Cosmology from random multifield potentials," JCAP 0603 (2006) 013, arXiv:hep-th/0512050 [hep-th].

[31] D. Marsh, L. McAllister, and T. Wrase, "The Wasteland of Random Supergravities," JHEP 1203 (2012) 102, arXiv:1112.3034 [hep-th].

[32] X. Chen, G. Shiu, Y. Sumitomo, and S. H. Tye, "A Global View on The Search for de-Sitter Vacua in (type IIA) String Theory," JHEP 1204 (2012) 026, arXiv:1112.3338 [hep-th].

[33] T. C. Bachlechner, D. Marsh, L. McAllister, and T. Wrase, "Supersymmetric Vacua in Random Supergravity," arXiv:1207.2763 [hep-th].

[34] Y. Sumitomo and S.-H. H. Tye, "A Stringy Mechanism for A Small Cosmological Constant," JCAP 1208 (2012) 032, arXiv:1204.5177 [hep-th].

[35] Y. Sumitomo and S.-H. H. Tye, "A Stringy Mechanism for A Small Cosmological Constant Multi-Moduli Cases -," arXiv:1209.5086 [hep-th].

[36] Y. Sumitomo and S.-H. H. Tye, "Preference for a Vanishingly Small Cosmological Constant in Supersymmetric Vacua in a Type IIB String Theory Model," Phys.Lett. B723 (2013) 406-410, arXiv:1211.6858 [hep-th].

[37] G. Borot, B. Eynard, S. Majumdar, and C. Nadal, "Large deviations of the maximal eigenvalue of random matrices," J.Stat.Mech. 1111 (2011) P11024, arXiv:1009.1945 [math-ph]. 